\title{
Monitoring the Experiment Process and Diagnosing the Experiment Mistakes Made by Students with Petri Net Modeling
}

\author{
Jyh-Cheng Chang ${ }^{1}$, Shao-Chun Li ${ }^{1}$, Maiga Chang ${ }^{2}$, and Jia-Sheng Heh ${ }^{1}$ \\ ${ }^{1}$ Chung-Yuan Christian University, Chung Li 32023, Taiwan \\ 2 National Science \& Technology Program for e-Learning, Chung Li 32023, Taiwan
}

\begin{abstract}
In this paper, Petri net theory is taken into analyzing the Virtual Experiment (VE) in the Virtual Experiment Environment (V.E.E.). After applied the Petri net to V.E.E., the V.E.E. then can monitor the experiment progress and measure the learning effects of students when the students are doing experiment. Petri net is an approach that can be used as a visual-communication aid similar to flow charts, block diagrams, and networks. Therefore, the experiment steps are easily modeled by Petri net. According to the data which is collected by the V.E.E., there are two major error types that a student might make during an experiment. The first error type is the procedural error and the other one is measuring error. In the end of this paper, an experiment system is implemented. There were $723^{\text {rd }}$ year students in junior high school involved in the experiment. In this experiment, we did both of pre-test and post-test in order to make sure the Petri net-enhanced V.E.E. is useful.
\end{abstract}

\section{VE, V.E.E., and Petri Net}

A Virtual Experiment Environment (V.E.E) in e-learning systems provides a visualized social learning environment by creating experiment situation on Internet. 7] [13] A set of simulated virtual equipments for students do experiment is also included in V.E.E.. 4 5] Recently, V.E.E. on WWW (World-Wide Web) is categorized as three major types, Real Lab, Virtual Lab and Visual Lab,

1. Real Lab: needs a set of external experiment devices and software kit. 6

2. Virtual Lab: is using a set of computer-simulated experiments. 1] 3

3. Visual Lab: is using video-media and some manipulation toolboxes to cover both reality and convenience purpose. [14]

According to the discussions of V.E.E., an interactive V.E.E. should consist of four major components, including teaching materials, user interfaces - UI (visual lab, in this paper), interactive protocols and knowledge base. As Fig [1] shown below, those four components operate cooperatively. 2] 9]

Learners do any scientific experiments as they like with appropriate visual lab interface (UI). An experiment agent (or agent, in shortly) and/or the system might be able to read the teaching materials directly without supervising, 


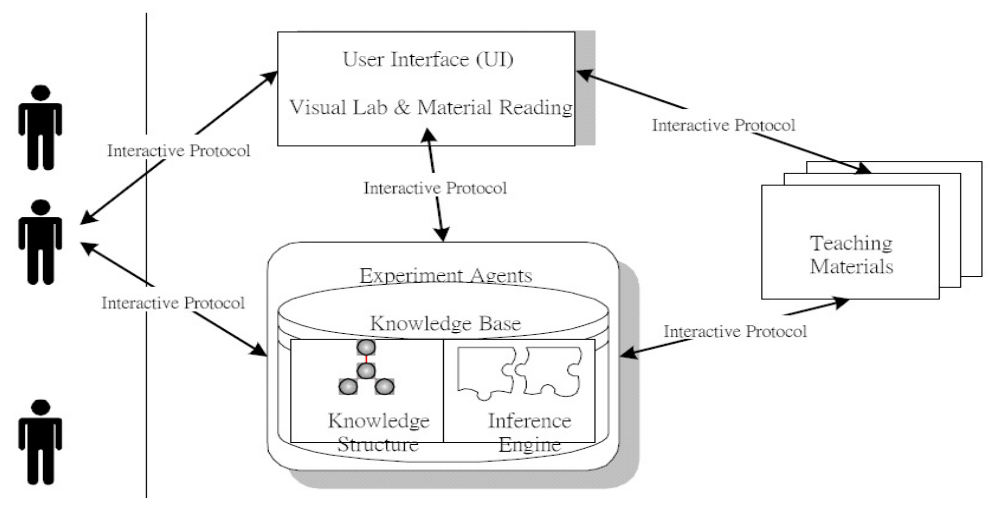

Fig. 1. Four components in V.E.E.

and store some well-organized knowledge (or information) into their artificial brains, which is so-called knowledge base. After understanding specific experiment, agent and/or the system owns capability to be a navigator while learners are doing labs.

Before we discuss how to use Petri net to analyze a virtual experiment, the process of designing a virtual experiment should be figured out first. Fig 2 shows a process of designing a VE. 12 .

First, we conducted the diagnostic test to grasp the intellectual level of what learners have already known. Second, an instruction design for introducing new knowledge to students is needed. The instruction design is composed of mutually related three stages - exploration, concept introduction, and concept application.

1. Learners in the exploration stage will find out the problem which they have been curious and try to solve the problem on their own.

2. Teachers in the concept introduction stage will put some concepts including what the learners have not learned yet, and the concept introduction stage is also the stage which is used to deal with the intellectual conflict.

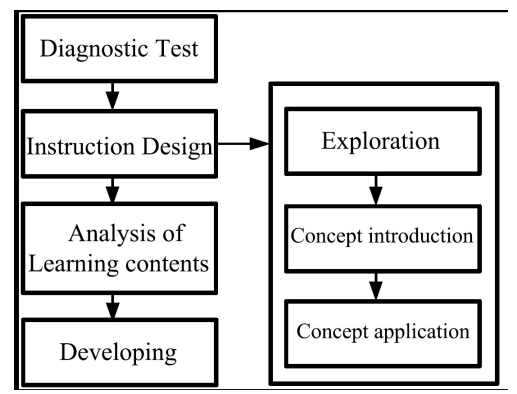

Fig. 2. A designing process for VE 
3. Teachers in the concept application stage will try to discover what has been learned by learners, and the concept application stage is also the stage which is used by learners to handle those similar real-life problems in future.

Third, the learning contents have been organized with three levels according to the degree of difficulty and been devised to put the learning subjects in a hierarchical structure. Finally, VE environments have been developed according to above steps. As mentioned in the previous Section, the VE is only doing operations and guidance. This study will try to use Petri net to solve the following three problems:

(a) How to record the experiment process of students to do the diagnosis?

(b) How to analyze the experiment procedures to model the virtual experiment?

(c) How to diagnose the mistakes that students make?

\section{Analyzing VE by Petri Net}

In order to solve the three problems proposed in previous Section, this paper applies Petri net with a self-developed knowledge instructions to the VE design. The knowledge instructions are the HCI (Human Computer Interaction) protocol which is a communication bridge between humans and computers. Furthermore, mapping the corresponding experiment steps to the basic elements of Petri net will make the comparisons between students and teacher more easily. By using Petri net and knowledge instructions the system (which is V.E.E. in this paper) will be able to identify four error types, including the procedural error, the record error, the estimate error, and tools error.

There are seven types of knowledge instructions. Each kind of knowledge instructions is formatted by XML. Therefore, the knowledge instructions can be used by the system to record the experiment changes and exchange information between experiment components and students. Furthermore, with XML-based knowledge instructions the system will be able to exchange information among experiment components either, no matter the experiment component is developed by what kind of technologies such as Flash, Java, or others. The sever types of knowledge instructions which are used for students to manipulate the virtual experiment are:

1. Load Instruction: which is used by students to load experiment tools/components.

2. Measure Instruction: which is used by students to gather the experiment data they want.

3. Select Instruction: which is used by students to take the experiment tools/components from the virtual experiment desk.

4. Release Instruction: which is happened when the students release the experiment tools/components.

5. Operate Instruction: which is used by student to manipulate the attributes of the experiment tools/components. 
6. Move Instruction: which is happened when student move the experiment tools/components by holding the left-key of the mouse down.

7. Record Instruction: which is used by students to store the data of the experiment tools/components after some kinds of measurement have done.

After applying the knowledge instruction to the V.E.E., the system will be able to record all operations which are manipulated by the student. More than that, once the operations can be integrated with Petri net and be mapped into virtual experiment, the standard procedure of a virtual experiment then could be understood by the system. Moreover, the system will have the ability of diagnosing the student's experiment procedure and providing the student manipulative demonstration and suitable hints. Fig 3 shows a complete Petri net-based virtual experiment example. The virtual experiment analyzed by Fig 3 is a Free-Falling experiment. This Free-Falling experiment contains two objects, one ball and one man.

The most important goal of this paper is to let the system (either the V.E.E. or the agent) have the ability of knowing what the student did and diagnosing the mistakes that the student made during the experiment. Once the system can understand the operations manipulated by the student according to the Petri net, the system will be able to classify the mistakes that the student made. The obvious error type is procedural error, because the standard procedure of doing the whole experiment is designed by Petri net. Besides the procedural error, the measuring errors could be discovered from the action logs.

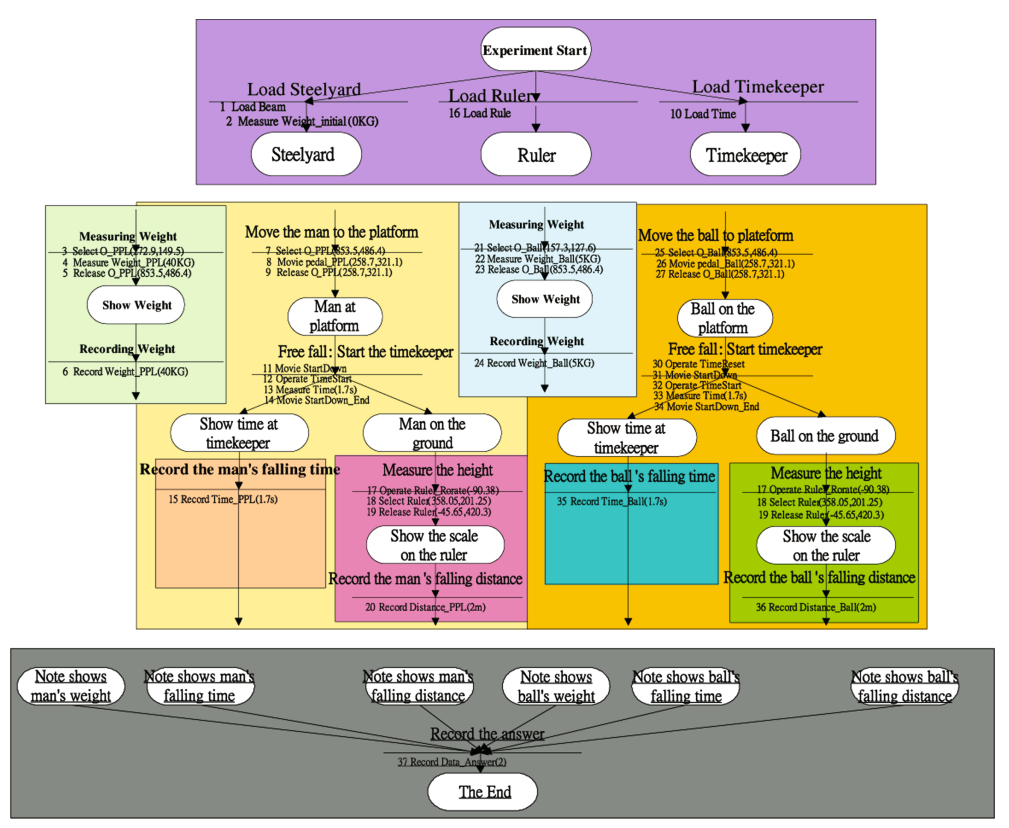

Fig. 3. A complete Petri net-based virtual experiment example 
1. Procedural Error: will happen when a student manipulated the experiment in one Petri net model (model A), for example, a state model or an action model, and then jump to another model (model B) before reaching the terminal of the original model (model A).

2. Measuring Error: can be divided into three kinds of errors

a Recording Error: will happen when the experiment data typed by the student is inconsistent with the real data recorded by the system automatically.

b Estimated Error: will happen when the answer calculated by the student is inconsistent with the real answer stored by the system.

c Tools Error: will happen when the student picked the wrong experiment tool. For example, if the experiment asks the student to take the angle, but the student pick a ruler up.

\section{Experiment System}

In this paper, a virtual experiment environment is developed. Within the V.E.E. there are some virtual experiments about the basic kinematics and could be used by the students at the junior high school level. Before we talk about the study results and discuss the collected data, the details of V.E.E. are described first. Since the virtual experiments in the V.E.E. are analyzed and developed with the Petri nets, the students' learning progresses and experiment mistakes then could be recorded and discovered by the system. The system's procedural diagram is just like the following (Fig 4):

Fig (5) (left) is a snapshot of the V.E.E., the virtual experiment that this figure shows is a free-falling experiment. There is a little girl stands on a platform. Her weight is about 40 kilograms (KG). Now, the experiment asks the student to do something for the experiment. However, the student might have no idea about what he/she should do. Therefore, the system provides a hint about the next step based on the standard experiment procedure which is developed by teacher with Petri net. As you can see there is a little person (or called an agent) stands on the ground at middle-bottom position in Fig 5 (left). The agent says in the dialogue bubble is "please load the tool, Timer".

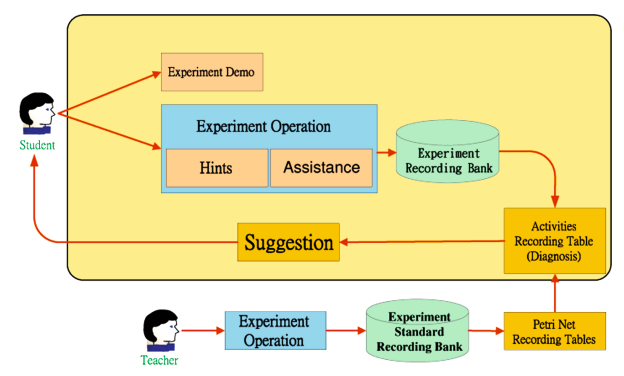

Fig. 4. The system's procedure diagram 


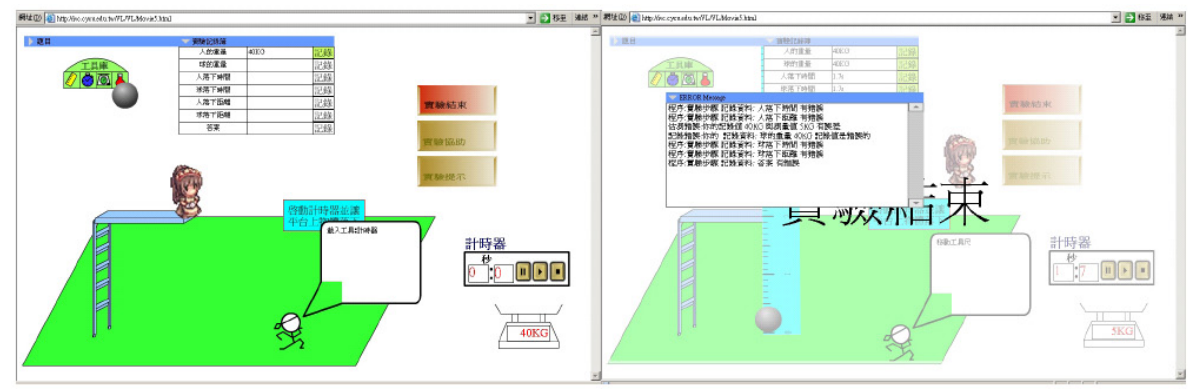

Fig. 5. The assistance of the agent in VE \& The diagnosis results

There three buttons in the V.E.E. as shown on the top-right corner in Fig 5 (left). The first red one is used by students to end the experiment. And the second one is to ask the assistance, the third one is to ask a hint. Fig 5 (right) is a snapshot with a diagnosis result just after the student completes the experiment. The V.E.E. pop-up an error message in order to tell the student what errors and mistakes that the students made during they did the experiment. The first four lines in the diagnosis result in Fig[5(right) are

1. procedural error: record of falling duration is incorrect

2. procedural error: record of falling distance is incorrect

3. estimated error: the weight that you enter is $40 \mathrm{KG}$ but the measurement shown on the weighting tool is $5 \mathrm{KG}$

4. recording error: the weight record is incorrect

\section{Results and Discussions}

In this study we cooperated with Mr. Lai who is a junior high school teacher. There were 3 classes students about 72 students participated this study. These participants were $3^{\text {rd }}$ year students which means it was their last year in the junior high school and their pre-knowledge should be enough. In this study all of students divided into three different groups, and we simply named each class as one group in order to make sure there was no any factor which might affect the study result. All students were asked to take pre-test before they did the virtual experiment and post-test after they finished the virtual experiment. The period of this study is June 10-17. The differences between the three groups in this study are listed by Table1.

As we can see in Table 2(left), the performance and improvement of these three groups is quite obviously. Although the average pre-test score of Group A was higher than Group B and C, the improvement made by the V.E.E. could be still easily told. Furthermore, the students in Group $\mathrm{C}$ got much more improvement with the Petri net-based virtual experiment.Besides the improvement differences between Group B and C, the errors which were made by students 
Table 1. Three Groups

\begin{tabular}{|r||c|c|c||}
\hline & Group A & Group B & Group C \\
\hline pretest-posttest & $\triangle$ & $\triangle$ & $\triangle$ \\
\hline using V.E.E & & $\triangle$ & $\triangle$ \\
\hline assists and hints & & & $\triangle$ \\
\hline
\end{tabular}

Table 2. Three Groups' progress \& Experiment Comparisons (average data)

\begin{tabular}{|c|c|c|c||c|c|c|}
\hline & Group A & Group B & Group C & & Group B & Group C \\
\cline { 2 - 4 } Pre-test & 61.16 & 51.04 & 52 & Procedural Errors & 5 & 5.625 \\
\hline Post-test & 61.21 & 56.16 & 58.08 & Measuring Errors & 14.92 & 10.75 \\
\hline Difference & 0.05 & 5.12 & 6.08 & Experiment Time & $25 \mathrm{~m}: 33 \mathrm{~s}$ & $21 \mathrm{~m}: 05 \mathrm{~s}$ \\
\hline
\end{tabular}

during the experiment could be also reduced by the Petri net-based virtual experiment. As Table 2(right) demonstrates, the average measuring error in group $\mathrm{C}$ is less than group B, which means the hints and assists that the system provided to students based on the Petri net-based virtual experiment was useful to the students. In another way, it is reasonable for the students in both of Group B and C made similar procedural errors. Because no matter which group one student belonged to, he/she did experiment in the same virtual experiment environment.

\section{Conclusions}

This paper applies Petri net analytical tools to the virtual experiment development. With Petri nets, a Virtual Experiment Environment or related agents will get the ability of providing the students hints and assists. Moreover, the system will be able to 'know' what the student is doing and the mistakes that the students probably had made during the experiment. Once the experiment procedure is well known by the system, the system can either demonstrate the whole experiment operation or show the student the next experiment step under the request which is made by the student.

\section{References}

1. M. Chang, I. Chung, Y.-W. Jeng and J.-S. Heh. A Cooperative and Visualized Environment for Virtual Experiment. International Computer Symposium, (ICS 1996), Kaohsiung, Taiwan, pages 16-23, 1996

2. M. Chang, R. Kuo and J.-S. Heh. Applying Navigation Mechanism to Virtual Experiment Environment on WWW with XML-style Teaching Materials. AACE World Conference on Educational Multimedia, Hypermedia \& Telecommunications, (ED-Media 2001), Tampere, Finland, Jun. 25-30, 2001, pages 257-262, 2001

3. I. Chung, M. Chang and J.-S. Heh. SoftLab: A Virtual Experiment Environment on WWW. The 6th International Conference on Computer-Assisted Instruction, (ICCAI 1997), Taipei, Taiwan, pages 220-223, 1997 
4. C.-W. Huang, C.-K. Hsu, M. Chang and J.-S. Heh. Designing an Open Architecture of Agent-based Virtual Experiment Environment on WWW. AACE World Conference on Educational Multimedia, Hypermedia \& Telecommunications, (ED-Media 1999), June 19-24, 1999, Seattle, WA, USA, pages 270-275, 1999

5. C.-W. Huang, S.-T. Sun, C.-C. Chang, K.-C. Jan and K.-Y. Yang. Education Software and Hardware. $2^{\text {nd }}$ Global Chinese Conference on Computer in Education, (GCCCE 1998), Hong-Kong, pages 50-57, 1998.

6. K.-C. Jan, Y.-W. Jeng, K.-Y. Yang and J.-S. Heh. Combined Real Experiment and Virtual Experiment on WWW. International Symposium on Multimedia Information Processing, Taipei, Taiwan, pages 521-527, 1997

7. Y-W. Jeng, M. Chang, I. Chung and J.-S. Heh. Designing Objects for Virtual Experiments. OOTA 1996, Tainan, Taiwan, pages 331-339, 1996

8. K. Jensen. "Colored Petri Nets - Basic concepts, analysis methods and practical use volume 1", Springer, second edition, 1996

9. R. Kuo, M. Chang and J.-S. Heh. Applying Interactive Mechanism to Virtual Experiment Environment on WWW with Experiment Action Language. IEEE First International Conference on Advanced Learning Technologies, (ICALT 2001), Madison, Wisconsin, USA, Aug. 6-8, 2001, pages 289-290, 2001

10. J. L. Peterson and E. Cliffs. Petri net theory and the modeling of systems. PrenticeHall, 1981

11. C. A. Petri. Kommunikation mit Automaten. Bonn: Institut fur Instrumentelle Mathematik, Schriften des IIM Nr. 2, 1962

12. Y.-S. Shin. "Virtual experiment environments design for science education" Cyberworlds, 2003. Proceedings, pp. 388-395, 3-5 Dec. 2003

13. S.-H. Tung. Visualizing Evaluation in Scheme. 1995

14. C.-C. Wang, M. Chang, C.-K. Hsu and J.-S. Heh. Visual Lab - A Multimedia Virtual Experiment Environment on WWW. AACE World Conference on Educational Multimedia, Hypermedia \& Telecommunications, (ED-Media 2000), Montreal, Quebec, Canada, Jun. 25- Jul. 1, 2000, pages 1509-1510, 2000 